\title{
ELECTRONIC RECORDER OF DEVIATION FROM STRAIGHTNESS WITH OPTICAL-LIGHT GUIDE ANALYZER
}

\author{
Zaxidov Nematjon ${ }^{1}$, Rahmatillayev Shahzod ${ }^{1}$, Gafurov Doniyor ${ }^{1}$ \\ ${ }^{1}$ Tashkent State Technical University, Department of Alternative Energy Sources, University str-2, \\ Tashkent, 100069, Uzbekistan
}

\begin{abstract}
The article discusses a technical solution aimed at improving the means of alignment measurements, based on measuring the deviation from straightness relative to the reference line set by the laser beam using a recorder, which uses a fiber-optic analyzer with a scanning shutter and an electronic block circuit that provides an increase in the measurement range when maintaining the minimum overall dimensions of the mass and energy intensity of the optoelectronic recorder with high efficiency in obtaining information on linear displacements.
\end{abstract}

\section{Introduction}

In the conditions of seismically active regions, where the time factor is often decisive, increased requirements are imposed on the reliability, durability, and safety of buildings and structures, including hydraulic structures. Accordingly, the requirements for the methods and means of geodetic control over their operation, including continuous monitoring, are increasing.

The practice of producing engineering and geodetic measurements when observing precipitation and deformations of engineering structures is based mainly on the use of visual-optical instruments, which, by their design and principle of operation, often do not provide the necessary efficiency, information content and productivity of the measurement process, are characterized by high labor intensity, require participation of a large number of performers to ensure the required accuracy [3].

Today, the main direction of improving the technology of engineering and geodetic measurements is the introduction of methods and tools based on the use of laser, infrared and other radiation sources in conjunction with photoelectric and optoelectronic recording devices, and allowing you to create fundamentally new methods and means of automation $[1,2,6]$.

For the automation of geodetic measurements, ray and interference lines were used as reference (reference) lines in alignment measurements. The alignment measurement method consists of determining perpendicular linear offsets from a given reference plane, although measurements are often taken relative to a single reference line. The following methods of alignment measurements have already become classical.
The diffraction method according to Yunga's scheme [2] provides a high measurement accuracy, of the order of 30-40 mkm, however, the need for an operator to visually observe the diffraction pattern on the screen limits the automation process; another disadvantage of the method is the small range of the alignment (up to $80-100 \mathrm{~m}$ ).

The dispersion method based on the Fraunhofer diffraction theory provides a medium range and high accuracy; the disadvantage is the complexity of the automation process.

The interference method has a large, about $1000 \mathrm{~m}$, range, high accuracy, and allows you to fully automate the measurement process, but high requirements for the accuracy of manufacturing and use of zone plates, as well as the influence of chromatic and spherical aberration, limit its application $[2,6]$.

The classical methods were replaced by laser emitters that appeared in the 60 s of the last century, which aroused interest from instrument manufacturers due to a number of exceptional optical properties: coherence (monochromaticity, singlephase), low consumption, concentration of light energy in the form of a beam, focusing by a plane by a wave front with collimating optics, on the basis of which emitters for alignment measurements are created, in which only the energy and geometric strict shape of the laser beam section is used [5,7].

The advantage of the photo sensors and differential type photo sensors used as recorders, based on the amplitude comparison method, is the absence of moving elements in it, but the disadvantages include: a limited measurement range due to the dependence of the optical channel (lens diameter) and the size of photo sensors (photo resistors, photodiodes, phototransistors, etc. others) 
[7]; limited measurement accuracy (zero drift, temperature, heterogeneity). Along with differential sensors, CCD structures, devices have been developed using mechanical scanning units that provide high accuracy, a wide range of measurements, the operation of which is based on counting the number of pulses depending on the time (time-pulse) angle rotation (angle-pulse) and linear ones with a moving shutter [1].

Analysis of measuring instruments $[1,2,3]$ has led to the need to develop a photo electronic measuring device, which excludes the use of a mirror with a rotating drum, i.e. devices with minimal dimensions, weight, high accuracy and low power consumption.

According to the authors, a fiber-optic analyzer with an ordered hexagonal stacking of fiber bundles at the input and output ends can be used for this purpose. The main optical characteristics of LEDs are: light transmission, numerical aperture - the maximum angle of incidence of transmitted light, refractive indices of the core and cladding, resolution and image contrast [4].

The authors have developed an optical-electronic recorder of deviation from straightness with a fiberoptic analyzer. The technical solution is registered with the Agency for Intellectual Property of the Republic of Uzbekistan, No. FAP 2019.0185 and is aimed at improving the means of alignment measurements and is based on measuring the deviation from straightness relative to the reference line set by the laser beam using a recorder that uses a fiber-optic analyzer with a scanning shutter and an electronic block diagram, providing an increase in the measurement range while maintaining the minimum overall dimensions of the mass and energy intensity of the optoelectronic recorder with high efficiency of obtaining information on linear displacements.

Unlike known analyzers, the electronic circuitry of the device operates on a slope-minimum signal.

Scanning using a flexible steel cable instead of a screw mechanism can reduce the weight of the device. The proposed device allows the use of a cable with a diameter of $0.5 \mathrm{~mm}$, while, taking into account the square dependence of the area on the radius, the mass of the cable and pulleys 4 will be only 10 grams.

Reducing energy consumption is achieved by reducing the amount of friction in the bearings of the drum with a screw slot.

The worm gear simultaneously plays the role of both a reducer and a mechanical converter of rotary motion into a linear one, which gives a clear advantage over a linear screw mechanism with an extended screw, depending on the measuring range. The introduced spring makes it possible to exclude errors in the linear expansion of the cable.

"Angle-pulse" converters have a great advantage over time-to-pulse converters, since they do not require stability and can operate at any, even changing speed.

If the width of the slot is $n=0.5 \mathrm{~mm}$, the length is $1=200 \mathrm{~mm}$, then the cross-sectional area is $\mathrm{S}=100$ $\mathrm{mm} 2$. We obtain the radius of the output part of the fiber bundle as

$$
\mathrm{R}=\sqrt{\mathrm{S}} / \pi=\sqrt{ } 100 / 3,14=5,6 \mathrm{~mm},
$$

respectively, the diameter is $11 \mathrm{~mm}$.

As can be seen from the calculations, an increase in the measurement range 1 does not lead to a cubic dependence, in contrast to the analogue, which makes it possible to sharply reduce the overall dimensions of the device.

It is possible to reduce the amount of backlash (screw and gear) of the worm drive, which affects the accuracy of measurements, by introducing a spring that works to reduce the distance between them, but while maintaining the minimum required coefficient of friction. Compared to $600 \mathrm{~mA}$ for the analog, the current consumption of the device is $\sim 65 \mathrm{~mA}$ due to the use of a lightweight worm drive.

The proposed device can find practical application in the production of high-precision engineering and geodetic measurements at engineering infrastructure facilities, in the installation and alignment of the rows of high-voltage transmission line supports, as well as in the installation and installation of an array of solar stations.

\section{Conclusion}

The proposed device can find practical application in the production of high-precision engineering and geodetic measurements at the facilities of the engineering infrastructure, in the installation of rows of an array of solar stations and solar equipment.

\section{References}

1. Zasarinniy A.V. Automation of high-precision engineering and geodetic measurements-M.:Nedra 1976.-247p

2. Yambayev X.K. Special devices for engineering and geodetic works-M.:Nedra 1990.-267 p.

3. Yeliseyev S.B. Geodetic instruments and devices-M.:Nedra, 1973.

4. Applied optics N.M.Zakasnova.M.:Mashinostroeniye, 1988.

5. A.s. SU №1670403 "Photovoltaic trip measuring device" Avt. N.M.Zaxidov application 12.12.1988., printed 1991 у. BI №30G01B21/00

6. Gauf .M. Elektronnie teodoliti I Taxiometri. Per.p. cheyskogo .-M.:Nedra, 1978.

7. A.s SU №1474466 «Fotoelektricheskoe ustroystvo dlya izmereniya lineynix razmerov ». Avt. Захидов, print. 1989, BI №15 G01B21/00. 